\title{
Implementation of Surface Gratings for Reduced Coupling Noise in Silicon-on-Insulator Circuits
}

\author{
Sam Werquin, Diedrik Vermeulen, and Peter Bienstman
}

\begin{abstract}
Coupling light into a silicon-on-insulator photonic chip has always been the first hurdle to overcome when using photonic integrated circuits. For applications that require robust, low-noise performance, and high degree of multiplexing, flood illuminating an array of vertical grating couplers is a promising approach to couple input light into the chip waveguides. This technique provides a very high alignment tolerance and allows simultaneous excitation of multiple waveguides for rapid parallel readout. However, parasitic reflections of the coupled light on the chip substrate introduce interferences and limit the device performance. We investigate the use of grating structures implemented on the chip surface to limit these parasitic signals and demonstrate a significant reduction of the coupling noise.
\end{abstract}

Index Terms-Photonic integrated circuits, interference, grating.

\section{INTRODUCTION}

$\mathbf{S}$ ILICON-ON-INSULATOR (SOI) photonic integrated circuits have proven to be an excellent platform for a variety of integrated applications like photonic biosensing [1], [2], optical coherence tomography [3] or on-chip laser Doppler vibrometry [4]. All of these are applications which are extremely sensitive to interference noise caused by parasitic paths. Therefore, low-noise performance combined with ease of use and robustness against external disturbances is a mandatory requirement for these devices. Rather than coupling light into the microchip waveguides using traditional fi er coupling techniques with vertically coupling gratings [5] which require delicate alignment and micrometer accuracy, one can make use of a collimated laser beam to illuminate the chip surface. This improves robustness against vibrations and allows for simultaneous excitation of a compact grating coupler array for better multiplexing possibilities [6]. However, various refections from the SOI deteriorate the device performance. Improved grating couplers for photonic applications [7], [8] and reflecti nless multi-mode interferometers [9] have been suggested. But parasitic transmission paths

Manuscript received March 21, 2014; revised May 9, 2014; accepted May 21, 2014. Date of publication May 23, 2014; date of current version July 24, 2014. This work was supported in part by the Special Research Fund, Ghent University, in part by the FP7 Project CanDo, and in part by the Innovative Medicines Initiative (RAPP-ID Project, Grant 115153).

S. Werquin and P. Bienstman are with the Center for Nano- and Biophotonics, Department of Information Technology, imec, Ghent University, Ghent B-9000, Belgium (e-mail: sam.werquin@intec.ugent.be; peter.bienstman@ugent.be).

D. Vermeulen is with Acacia Communications, Hazlet, NJ 07730 USA (e-mail: diedrik.vermeulen@acacia-inc.com).

Color versions of one or more of the f gures in this letter are available online at $\mathrm{http}: / /$ ieeexplore.ieee.org.

Digital Object Identifie 10.1109/LPT.2014.2326735

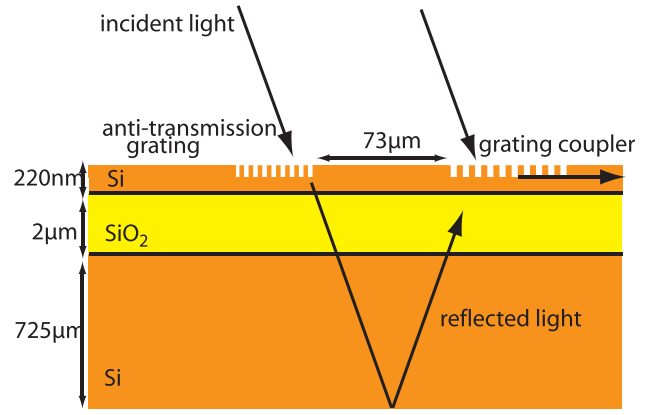

Fig. 1. Schematic representation of light incident on chip surface. Light coupling to the waveguide through the grating coupler after reflectio on the substrate induces an interference pattern. An anti-transmission grating can be implemented to block this parasitic light path.

through the chip substrate still form an important limitation of the device performance by inducing interferences in the coupled light. Here, we develop an integrated anti-transmission grating that further enhances the coupling to the integrated circuit. Simulation results and experimental demonstrations are reported.

\section{Surface Grating Structures}

Fig. 1 shows a schematic representation of the incident light and the layered microchip surface. The chip is processed from a Smart-Cut SOI wafer [10] and consists of a thick silicon substrate, a $2 \mu \mathrm{m}$ thick oxide buffer layer and a $220 \mathrm{~nm}$ silicon top layer. It is in this top silicon layer that integrated photonic structures such as microring sensors are patterned. The chip surface is illuminated by a collimated light beam with an incidence angle of $10^{\circ}$. Several waveguides can be excited simultaneously by a single input beam. The fraction of the light incident on a grating coupler [11] in a waveguide couples directly into the photonic wire waveguide with an eff ciency up to $30 \%$ and an angle tolerance below $1 \mathrm{~dB}$ per $2^{\circ}$. However, light incident on the chip surface in the area in front of this grating will be transmitted through the silicon-air interface. This beam propagates through the chip substrate and ref ects at the back surface. As the longitudinal component of the wavevector is conserved upon refraction, the Bragg condition (1) for the grating coupler is also met by the ref ected light beam.

$$
\beta=k_{\text {in }} \sin (\theta)+K
$$

In (1), $\beta=\frac{2 \pi}{\lambda} n_{\text {eff }}$ is the propagation constant of the guided mode with effective index $n_{\text {eff }}$ and $K=\frac{2 \pi}{\Lambda}$, with $\Lambda$ the 


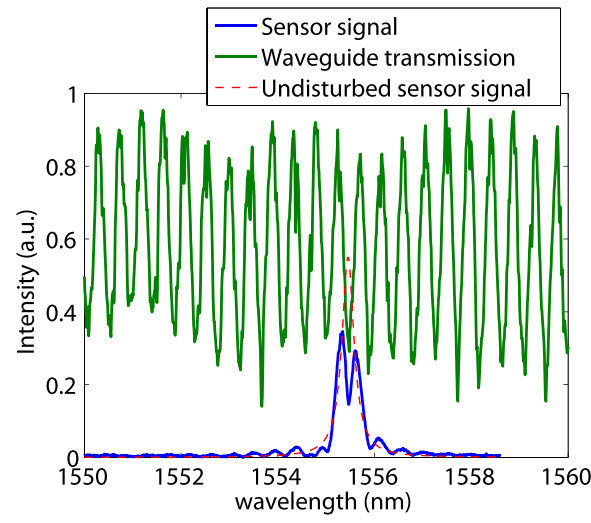

Fig. 2. Transmission signals disturbed by interference due to parasitic reflection. The dashed line shows the undisturbed sensor signal.

period of the grating. $k_{i n}=\frac{2 \pi}{\lambda}$ is the wavevector and $\theta$ is the angle between the incoming light and the surface normal, $\theta=10^{\circ}$ in this case. The ref ected light will then couple eff ciently through the burried oxide layer to the waveguide. The round trip through the chip substrate introduces a signif cant path length difference and affects the phase of the reflecte light. The phase difference between the directly coupled and the ref ected light generates a strong interference pattern in the input light. The effect on the transmitted power in the microchip for the application of microring resonator sensors is demonstrated in fig 2 , where it can be seen that the interference completely destroys the resonance line shape. The microring resonator used in this experiment consists of a $450 \mathrm{~nm}$ by $220 \mathrm{~nm}$ silicon wire waveguide. With a total round-trip length of $140 \mu \mathrm{m}$ and directional coupler spacing of $250 \mathrm{~nm}$, this results in a resonance line width of $200 \mathrm{pm}$ and a free spectral range of $3.6 \mathrm{~nm}$ in water.

The pattern has a period of $450 \mathrm{pm}$ and modulates the microring drop signals to an extent that the peak intensity is greatly suppressed and the sensor signal is effectively destroyed. Experimental characterization of the chip thickness conf rms that refections of the back surface generate an interference pattern with the observed period. Even in the case the microring signal is not completely destroyed, the interference pattern can still severely deteriorate sensing operation. During the detection of a biomolecule, the change in refractive index at the surface of the biosensor induces a shift in wavelength visible in the sensor signal. However, the strong modulation signal does not shift. This gives rise to highly undesirable features in an experimentally recorded binding curve and could lead to false detection events. Therefore, we suggest an integrated solution that can be implemented with the same lithographic procedure used for the definitio of the transducer and thus does not require any additional processing steps. We implement anti-transmission grating structures at the chip-air interface in the position that leads to the parasitic light path, as shown in fi. 1. This limits the transmission efficien $y$ and reduces the intensity of the reflecte light. Other techniques, like local metal deposition to absorb the parasitic light yield similar results, but this requires additional costly processing steps. We will show that our approach eff ciently

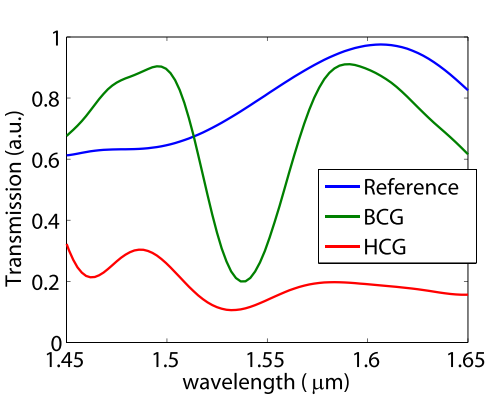

(a)

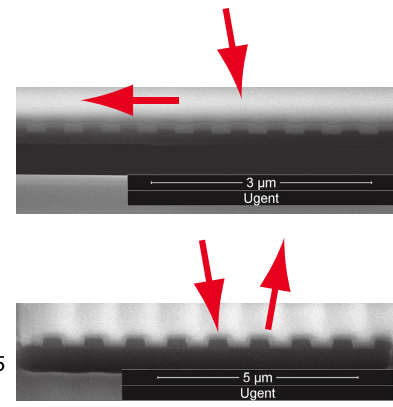

(b)
Fig. 3. (a) Simulated transmission to the substrate for different grating implementations. Introduction of the surface gratings significantl reduces the transmitted power. (b) Scanning electron micrograph of backward (top) and high-contrast grating (bottom) with schematic representation of light propagation.

reduces the parasitic reflecte signals and their corresponding interference pattern, without introducing additional processing steps.

\section{Simulations}

Using two-dimensional f nite-difference time-domain (2D-FDTD) simulations, we have investigated the feasibility of two different implementations for the anti-transmission grating of fg. 1. A f rst approach uses a backward-coupling grating (BCG) to diffract light to the waveguide in the opposite direction. This light will then propagate away from the grating coupler rather than refracting to the substrate region. Using a taper structure, the backward propagating light is dissipated in the silicon slab region that covers the chip surface. The grating is realized by etching $70 \mathrm{~nm}$ deep lines in the $220 \mathrm{~nm}$ thick waveguide layer, with a duty cycle of $50 \%$. For light with a wavelength of $1550 \mathrm{~nm}$ and an incidence angle of $10^{\circ}$, the Bragg condition implies a grating period of $540 \mathrm{~nm}$ for the BCG. Alternatively, we have also investigated the implementation of a high-contrast grating (HCG). Instead of guiding the light to the slab region, the HCG prevents light from reaching the grating coupler by maximizing the frst-order ref ection. This type of gratings has been primarily investigated for the applications in vertical-cavity surface-emitting lasers (VCSELs) because of its high refection over wide wavelength bands and relatively large fabrication tolerances [12], [13]. The high contrast is obtained by etching $220 \mathrm{~nm}$ deep lines through the full height of the waveguide layer. Fig. 3a shows the simulated transmission to the substrate for the reference situation and after implementation of the gratings. These simulations clearly show a signif cant reduction of the transmitted power after processing the gratings on the chip surface. A fundamental difference between both approaches also becomes apparent. The BCG is only eff cient in a narrow band around the design wavelength of $1550 \mathrm{~nm}$, whereas the HCG can introduce high refection over a relatively wide wavelength band. As described in [14], more complex simulation methods can be used to further optimize the HCG design. 


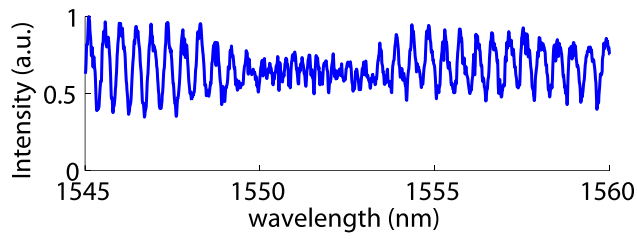

(a)

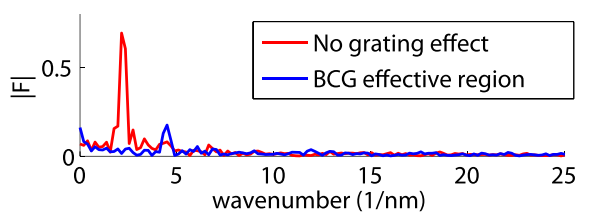

(c)

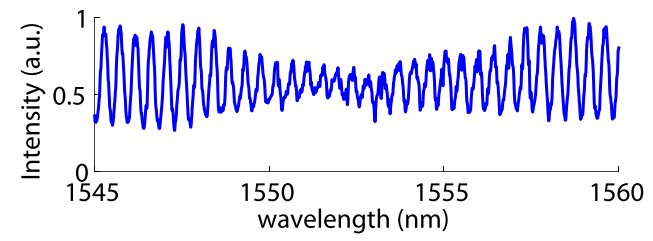

(b)

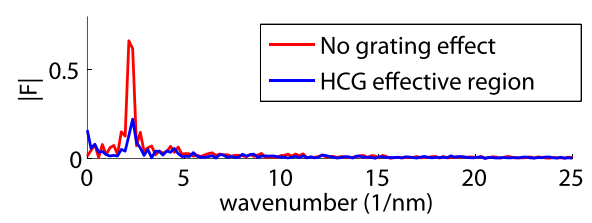

(d)

Fig. 4. Transmission spectra of grating coupler combined with BCG (a) and HCG (b). The grating effective region is clearly visible in both cases. (c) and (d) show the comparison of the Fourier spectrum in and outside the grating region of effect for BCG and HCG, respectively. The dominant Fourier components are signif cantly reduced in both cases.

\section{EXPERIMENTS}

Based on the simulation results, a design combining the grating couplers and the anti-transmission gratings has been fabricated in a complementary metal-oxide-semiconductor (CMOS) pilot line at imec. Scanning electron micrographs of the fabricated gratings are shown in fi . $3 \mathrm{~b}$, with red arrows to represent the direction of light propagation. The difference in etch depth and period of both gratings is clearly visible. For a chip thickness of $725 \mu \mathrm{m}$, the location of the gratings on the surface is calculated to be $73 \mu \mathrm{m}$ in front of the grating coupler. In this position, the designed gratings will prevent the incident light to be transmitted to the back of the substrate and reflecte up to the grating coupler. This eliminates the parasitic interferences. The chip is mounted on a measurement chuck for characterization. A collimated laser beam illuminates the input grating couplers and the surrounding chip surface. The tunable laser is swept at $5 \mathrm{~nm} / \mathrm{s}$ over a $20 \mathrm{~nm}$ range with a wavelength resolution of $10 \mathrm{pm}$. An infrared camera is used to record the output spectra with a frame rate of $500 \mathrm{~Hz}$.

\section{A. Results}

Fig. 4a shows the recorded spectrum for a grating coupler combined with a 35-period BCG with grating period of $530 \mathrm{~nm}$. Around $1551.5 \mathrm{~nm}$, a region with reduced interference effects is clearly visible. This suppression region corresponds to the grating wavelength targeted during design. The data are analysed by Fourier transforming the spectrum. This provides more insight into the origin of the oscillations. Fig. 4c compares the Fourier spectrum of a $3 \mathrm{~nm}$ region around $1551.5 \mathrm{~nm}$ to that of a $3 \mathrm{~nm}$ region with maximum interference strength. This shows the dominant component corresponding to oscillations with a $450 \mathrm{pm}$ period is successfully suppressed in the $1551.5 \mathrm{~nm}$ region. The suppression region shows the occurance of a new Fourier component corresponding to $220 \mathrm{pm}$ period oscillations, but with significa tly lower intensity. This component can be attributed to second-order ref ections of the chip substrate. Indeed, light incident on the chip surface $146 \mu \mathrm{m}$ in front of the grating coupler will make two round trips through the substrate, ref ecting twice off the bottom surface and thus arriving at the grating coupler with lower intensity and giving rise to less pronounced interference effects. Fig. $4 \mathrm{~b}$ and $4 \mathrm{~d}$ show the results for a 30-period HCG implemented $73 \mu \mathrm{m}$ in front of the grating coupler. The period of the HCG is $1050 \mathrm{~nm}$. Here, the $450 \mathrm{pm}$ period remains the dominant oscillation, while its strength is significa tly reduced. A small component at $220 \mathrm{pm}$ can barely be discerned from noise.

\section{B. Discussion}

The intensity pattern in the signal waveguide can be modeled as a simple two-beam interference signal given by

$$
I=I_{1}+I_{2}+2 \sqrt{I_{1} I_{2}} \cos (\Delta \phi)
$$

Here $I_{1}$ is the intensity of the light coupling directly into the waveguide through the grating coupler, $I_{2}$ is the intensity of the light coupling in the waveguide via refections on the backside and $\Delta \phi$ accounts for the path length difference between both interfering waves. As the Fourier components from Fig. 4c and fi . 4d originate from the only oscillating term of (2), they are proportional to the amplitude of the cosine term, or $2 \sqrt{I_{1} I_{2}}$. Comparing two Fourier spectra provides us with a means to determine the reduction in intensity of the parasitic beam $I_{2}$ as a consequence of the implementation of the surface grating structures. The intensity reduction or grating suppression eff ciency is given by

$$
10 \log _{10}\left[\left(\frac{X_{\text {ref }}}{X_{\text {eff }}}\right)^{2}\right]
$$

Here, $X_{\text {ref }}$ and $X_{\text {eff }}$ are the amplitude of the dominant Fourier components outside and inside the grating's spectral region of effect, respectively. For the $\mathrm{BCG}$, the signal originating from second-order ref ections becomes dominant in the suppressed region where the $450 \mathrm{pm}$ component is completely suppressed. Therefore, the strength of the Fourier component corresponding to $220 \mathrm{pm}$ period oscillations should be used for $X_{e f f}$ in (3). For the HCG, the $450 \mathrm{pm}$ component remains 


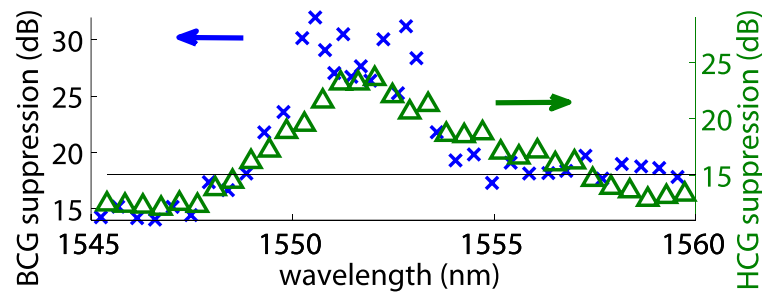

Fig. 5. Wavelength dependency of the ratio $I_{1} / I_{2}$ for the high-contrast and backward-coupling gratings. The suppression threshold is indicated by a line. A higher maximum suppression is obtained for the BCG, but the HCG is effective over a wider wavelength range.

dominant, so $X_{\text {eff }}$ corresponds to the Fourier component of this period. This results in an efficie cy of $12 \mathrm{~dB}$ for the BCG and an eff ciency of only $10 \mathrm{~dB}$ for the HCG. The obtained eff ciencies are only representative for the grating effect around the central grating wavelength. To obtain a spectral representation of the grating eff ciency, a different model can be applied. The strength of the interference pattern is also apparent in the extinction ratio of consecutive fringes. From (2), the dependence of the ratio $I_{1} / I_{2}$ on the extinction ratio is analytically calculated to be

$$
\sqrt{I_{1} / I_{2}}=\frac{(1-x)}{-(1+x)+\sqrt{4 x}}
$$

with $x$ the intensity ratio between consecutive maxima and minima of the spectrum. The result is plotted in $\mathrm{fg}$. 5 , both for the BCG and the HCG.

Outside the grating effective region, we can determine a baseline level. Without grating effect, $I_{2}$ is found to be 12 to $15 \mathrm{~dB}$ weaker than the intensity of light coupling directly into the waveguide. We experimentaly $\mathrm{f}$ nd an extinction due to the BCG up to $27 \mathrm{~dB}$, or $12 \mathrm{~dB}$ higher than the baseline. Here, we have to keep in mind that this number is limited by secondorder ref ections of the chip substrate. The direct transmission through the grating is practically completely eliminated, as suggested by the Fourier spectrum. If we set an arbitrary suppression threshold to an additional extinction of $3 \mathrm{~dB}$, the bandwidth of the suppressed region is $6 \mathrm{~nm}$, with a $4 \mathrm{~nm}$ region that exceeds $10 \mathrm{~dB}$ suppression. For the HCG, we calculate a reduction of the intensity $I_{2}$ up to $24 \mathrm{~dB}$, or a suppression of $11 \mathrm{~dB}$. The maximum suppression is reduced compared to that of the BCG, but the suppression threshold is exceeded over a wider range, i.e. $8 \mathrm{~nm}$. As the free spectral range of the microring sensors is $4 \mathrm{~nm}$, we can always f nd a microring resonance inside the suppression regions. We notice the suppression eff ciencies obtained from the spectral representation correspond very well to values calculated from the Fourier components. The difference between the simulation and the experiment for the HCG can be attributed to the simulation model, which is less suited for describing the very fast phase changes in the high-contrast region and the design parameters are not accurately predicted. The results discussed in this paragraph suggest the BCG as the better solution for the interference problem. If this grating is repeated in the position leading to the second-order ref ections, additional performance improvements are expected.

\section{CONClusion}

For ease of use, the delicate fber-chip interface should be avoided in complex integrated devices for a variety of applications. Instead, a collimated laser beam can be used for a robust coupling method with high alignment tolerance. In this letter, we suggested a straightforward technique to reduce parasitic substrate reflecti ns inherent to this approach. We demonstrated that implementing anti-transmission gratings to block parasitic light paths eff ciently reduces coupling induced interferences, without additional processing steps. In particular, a high-contrast ref ection grating and a grating that diffracts incoming light in the backward propagation direction of the waveguide were investigated. A suppression eff ciency of the coupling noise up to $12 \mathrm{~dB}$ was obtained for the backward-coupling grating.

\section{REFERENCES}

[1] M. Iqbal et al., "Label-free biosensor arrays based on silicon ring resonators and high-speed optical scanning instrumentation," IEEE $J$ Sel. Topics Quantum Electron., vol. 16, no. 3, pp. 654-661, Jun. 2010.

[2] A. J. Qavi and R. C. Bailey, "Multiplexed detection and label-free quantitation of microRNAs using arrays of silicon photonic microring resonators," Angew. Chem., vol. 49, no. 27, pp. 4608-4611, Jun. 2010.

[3] G. Yurtsever, B. Považay, A. Alex, B. Zabihian, W. Drexler, and R. Baets, "Photonic integrated Mach-Zehnder interferometer with an on-chip reference arm for optical coherence tomography," Biomed. Opt. Exp., vol. 5, no. 4, p. 1050, Mar. 2014.

[4] Y. Li, P. Segers, J. Dirckx, and R. Baets, "On-chip laser Doppler vibrometer for arterial pulse wave velocity measurement," Biomed. Opt. Exp., vol. 4, no. 7, pp. 1229-1235, Jul. 2013.

[5] G. Roelkens, D. Vermeulen, R. Selvaraja, W. Bogaerts, and D. V. Thourhout, "Grating-based optical f ber interfaces for siliconon-insulator photonic integrated circuits," IEEE J. Quantum Electron., vol. 17 , no. 3, pp. 571-580, Jun. 2011

[6] K. D. Vos et al., "Multiplexed antibody detection with an array of silicon-on-insulator microring resonators," IEEE Photon. J., vol. 1, no. 4, pp. 225-235, Oct. 2009.

[7] Y. Li, D. Vermeulen, Y. D. Koninck, G. Yurtsever, G. Roelkens, and R. Baets, "Compact grating couplers on silicon-on-insulator with reduced backref ection," Opt. Lett., vol. 37, no. 21, pp. 4356-4358, Nov. 2012.

[8] D. Vermeulen et al., "Ref ectionless grating couplers for silicon-oninsulator photonic integrated circuits," Opt. Exp., vol. 20, no. 20, pp. 1919-1921, 2012.

[9] Y. Li and R. Baets, "Improved multi-mode interferometers (MMIs) on silicon-on-insulator with the optimized return loss and isolation," in Proc. Annu. Symp. IEEE Photon. Benelux Chapter, 2011, pp. 205-208.

[10] B. Aspar et al., "Basic mechanisms involved in the smart-cut ${ }^{\circledR}$ process," Microelectron. Eng., vol. 36, no. 1, pp. 233-240, 1997.

[11] F. V. Laere et al., "Compact and highly eff cient grating couplers between optical fibe and nanophotonic waveguides," J. Lightw. Technol., vol. 25 , no. 1, pp. 151-156, Jan. 2007.

[12] M. C. Huang, Y. Zhou, and C. J. Chang-Hasnain, "A surface-emitting laser incorporating a high-index-contrast subwavelength grating," Nature Photon., vol. 1, no. 2, pp. 119-122, Feb. 2007.

[13] Y. Zhou, M. C. Y. Huang, and C. J. Chang-Hasnain, "Large fabrication tolerance for VCSELs using high-contrast grating," IEEE Photon. Technol. Lett., vol. 20, no. 6, pp. 434-436, Mar. 15, 2008.

[14] V. Karagodsky, F. G. Sedgwick, and C. J. Chang-Hasnain, "Theoretical analysis of subwavelength high contrast grating reflector ." Opt. Exp., vol. 18 , no. 16, pp. 16973-16988, Aug. 2010. 